\title{
ON THE REDSHIFT OF THE VERY HIGH ENERGY BLAZAR 3C 66A
}

\author{
A. Furniss ${ }^{1}$, M. Fumagalli ${ }^{2,3,6}$, C. Danforth ${ }^{4}$, D. A. Williams ${ }^{1}$, and J. X. Prochaska ${ }^{5}$ \\ ${ }^{1}$ Santa Cruz Institute of Particle Physics and Department of Physics, University of California Santa Cruz, 1156 High Street, Santa Cruz, CA 95064, USA \\ ${ }^{2}$ Carnegie Observatories, 813 Santa Barbara Street, Pasadena, CA 91101, USA \\ ${ }^{3}$ Department of Astrophysics, Princeton University, Princeton, NJ 08544-1001, USA \\ ${ }^{4}$ CASA, Department of Astrophysical and Planetary Sciences, University of Colorado, 389-UCB, Boulder, CO 80309, USA \\ ${ }^{5}$ Department of Astronomy and Astrophysics, UCO/Lick Observatory, University of California, 1156 High Street, Santa Cruz, CA 95064, USA \\ Received 2013 January 21; accepted 2013 February 8; published 2013 March 5
}

\begin{abstract}
As a bright gamma-ray source, $3 \mathrm{C} 66 \mathrm{~A}$ is of great interest to the high-energy astrophysics community, having a potential for placing cosmological constraints on models for the extragalactic background light (EBL) and the processes which contribute to this photon field. No firm spectroscopic redshift measurement has been possible for this blazar due to a lack of intrinsic emission and absorption features in optical spectra. We present new far-ultraviolet spectra from the Hubble Space Telescope/Cosmic Origins Spectrograph (HST/COS) of the BL Lac object 3C 66A covering the wavelength range 1132-1800 A. The data show a smooth continuum with intergalactic medium absorption features which can be used to place a firm lower limit on the blazar redshift of $z \geqslant 0.3347$. An upper limit is set by statistically treating the non-detection of additional absorbers beyond $z=0.3347$, indicating a redshift of less than 0.41 at $99 \%$ confidence and ruling out $z \geqslant 0.444$ at $99.9 \%$ confidence. We conclude by showing how the redshift limits derived from the COS spectra remove the potential for this gamma-ray emitting blazar to place an upper limit on the flux of the EBL using high energy data from a flare in 2009 October.
\end{abstract}

Key words: BL Lacertae objects: individual (3C 66A) - diffuse radiation - intergalactic medium - ultraviolet: general

Online-only material: color figure

\section{INTRODUCTION}

Direct measurements of the extragalactic background light (EBL) are difficult due to strong foreground sources in our solar system (Zodiacal light) and the Galaxy (Hauser \& Dwek 2001). If a direct measurement were possible, it would only reflect the current integrated state, leaving still the model-dependent task of extracting the time evolution. These difficulties have been overcome through the use of extragalactic very high energy (VHE, $E \geqslant 100 \mathrm{GeV}$ ) gamma rays from blazars with known redshifts (e.g., Aharonian et al. 2006; Mazin \& Raue 2007; Albert et al. 2008; Gilmore et al. 2009; Orr et al. 2011), the most commonly detected type of VHE extragalactic source. A blazar is a type of active galactic nucleus (AGN) that has a jet pointed toward the observer, and exhibits a highly polarized broadband spectrum from beamed, non-thermal emission processes.

The energy-dependent absorption of gamma rays by the EBL softens the intrinsic VHE gamma-ray spectra emitted by extragalactic objects. The details of the absorption depend on the shape of the EBL spectral energy distribution (SED) in the near-IR to optical band. Additionally, the total power and the shape of the SED of the EBL is shown to vary strongly with redshift in the currently available models, such as Dominguez et al. (2011), Gilmore et al. (2012), Kneiske \& Dole (2010), and Finke et al. (2010). To correctly account for the gamma-ray absorption, an accurate redshift of the VHE extragalactic target is required.

Approximately one-third of the current VHE extragalactic catalog ${ }^{7}$ is made up by blazars at unknown or poorly constrained redshift. 3C 66A is one of these blazars, with an uncertain spectroscopic redshift based on possible corroborating

\footnotetext{
6 Hubble Fellow.

7 http://tevcat.uchicago.edu/
}

measurements of single lines (Miller et al. 1978; Kinney et al. 1991; Bramel et al. 2005). Despite multiple attempts, in particular two high signal-to-noise ( $\mathrm{S} / \mathrm{N})$ measurements using Keck I (shown in Figure 1), no solid spectroscopic measurement based on the detection of multiple lines from the host galaxy has been possible. The lack of spectral features is not surprising given that 3C 66A is a BL-Lac-type-object AGN that, by definition, displays weak or no lines.

To overcome the inherent featureless characteristic of the $3 \mathrm{C} 66 \mathrm{~A}$ optical spectrum and enable deabsorption of the VHE spectrum with reliable redshift information, we have determined limits on the redshift of the blazar through the observation and statistical analysis of far-UV (FUV) absorption by the low- $z$ intergalactic medium (IGM). This method, already applied to the VHE blazars PG 1553+113 and S5 0716+714 (Danforth et al. 2010, 2013), sets a redshift lower limit using absorption lines caused by the intervening IGM. Further, given the expected distribution of IGM absorbers as a function of redshift, one can model any lack of absorption lines at longer wavelengths to statistically infer an upper limit on the blazar redshift.

\section{OBSERVATIONS AND SPECTRAL ANALYSIS}

3C 66A was observed with the Cosmic Origins Spectrograph (COS) during two epochs as part of two different programs. The blazar was observed for five Hubble Space Telescope (HST) orbits on 2012 November 1, with the medium resolution G130M grating COS/G130M (1135 $₫<\lambda<1450 \AA, 15.3 \mathrm{ks})$ as part of program 12621 (PI: Stocke). Three more orbits were devoted to observations with the COS/G160M $(1400 \AA<\lambda<1795 \AA$, $7.2 \mathrm{ks}$ ) grating under program 12863 (PI: Furniss) on 2012 November 8 . The calibrated, one-dimensional spectra for each exposure were obtained from the Mikulski Archive for Space Telescopes. 


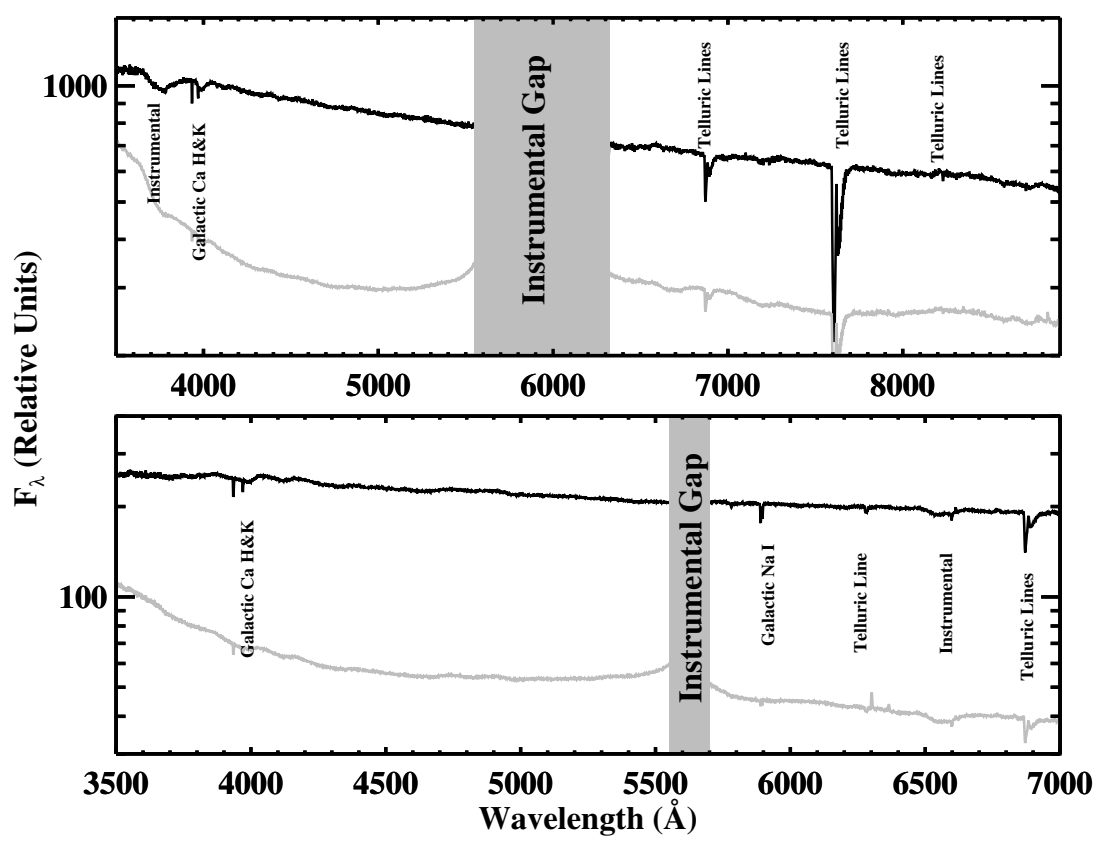

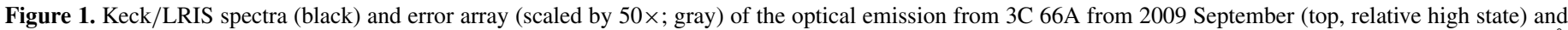

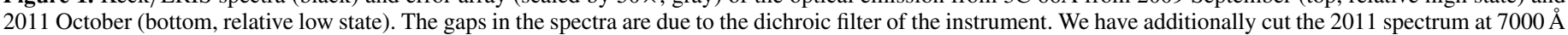

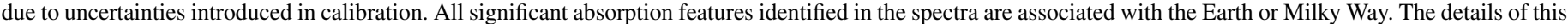

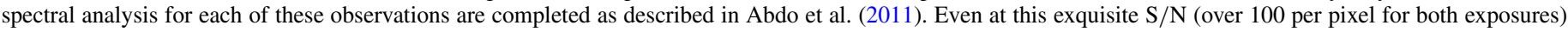
there are no features with which to place a constraint on the redshift of this blazar.

The G130M data show a flux mismatch between the shortand long-wavelength segment of each exposure and a $8 \%$ correction is applied to each before coaddition to bring them into the expected smooth continuum. The G160M data are considerably noisier; no flux discrepancy was observed and no correction was undertaken. The corrected exposures were then coadded with the standard IDL procedures described in detail by Danforth et al. (2010). This procedure includes an automatic scaling of the exposures taken during different epochs. The continuum flux level appears to have varied by $\lesssim 10 \%$ during the week between observing epochs, well within the current flux calibration uncertainty.

The combined spectrum continuously covers the wavelength interval 1132-1800 $\AA$, and shows the expected smooth continuum and narrow absorption features. The data quality varies over the spectral range due to the different sensitivities and exposure times in the two gratings. The mean $\mathrm{S} / \mathrm{N}$ per pixel in the continuum is $\sim 10(\sim 5)$ with nominal dispersions of $9.97 \mathrm{m \AA} \mathrm{pixel}{ }^{-1}$ (12.23 $\AA$ pixel $\left.{ }^{-1}\right)$ in the G130M (G160M) portion of the spectrum. $\mathrm{S} / \mathrm{N}$ values per 7 pixel resolution element are approximately twice these values (see Keeney et al. 2012). For additional details on the COS instrument, see Ghavamian et al. (2010).

Detailed analysis of these data and of the intervening absorption line systems will be presented in C. W. Danforth et al. (in preparation). In this paper, we exclusively focus on the spectral features that are useful to constrain the unknown redshift of the blazar $\left(z_{\text {blazar }}\right)$. The goal of the following analysis is to use absorption lines that arise from gas clouds in the IGM to establish a firm lower limit on the distance to $3 \mathrm{C} 66 \mathrm{~A}$ and to set an upper limit for the blazar redshift based on a statistical argument.

A visual inspection of the spectrum reveals the presence of multiple absorption systems for which both Lyman $-\alpha$ and Lyman $-\beta$ (Ly $\alpha$ and $\operatorname{Ly} \beta$ ) lines are detected. Among those, we identify three clouds at $z_{\mathrm{abs}} \sim 0.3283,0.3333$, and 0.3347 (see
Figure 2). All other lines detected at $>4$ sigma significance redward of these three Ly $\alpha$ systems are identified as Milky Way absorption (see Figure 3). Thus, because of the presence of a system at $z=0.3347$, we set a secure redshift lower limit of $3 \mathrm{C} 66 \mathrm{~A}$ at $z_{\text {blazar }} \geqslant z_{11}=0.3347$. We also search the spectrum for OVI $(\lambda \lambda 1031,1037)$ doublets that, owing to their bluer rest-frame wavelengths, could yield a more stringent redshift lower limit than the one set by absorption in the Lyman series. However, we do not find any instances of absorption beyond $z \sim 0.33$.

Next, we can exploit the lack of absorption beyond $z_{11}=$ 0.3347 to set an upper limit $z_{\mathrm{ul}}$ to the blazar redshift following a statistical argument. The frequency of absorption lines arising from the Lyman forest in the local universe has been measured along sightlines to extragalactic sources by different authors (e.g., Penton et al. 2004; Danforth \& Shull 2008). It is common to express this quantity with the function $d N\left(W>W_{0}\right) / d z$ which describes the average number of absorption lines with rest-frame equivalent width in excess to $W_{0}$ per unit redshift. We can therefore estimate the number of lines we expect to detect between $z_{11}$ and $z_{\mathrm{ul}}$, given the rest-frame limiting equivalent width $W_{\text {lim }}$ of the COS spectrum. By comparing the predicted number of absorption lines in a given redshift interval with the lack of detection beyond $z_{11}=0.3347$, we obtain redshift upper limit.

First, we generate 1000 mock spectra in the observed wavelength range 1215-1800 A by drawing Lyman forest lines from a distribution as a function of redshift such that the number of lines satisfies the observed $d N\left(W>W_{0}\right) / d z$. In this analysis, we assume no evolution in Ly $\alpha$ forest line incidence and adopt the frequency distribution from Danforth \& Shull (2008), although a similar result is obtained if we adopted the distribution from Penton et al. (2004). Next, we assign to each line a Doppler parameter drawn from the observed distribution in the local IGM (Danforth \& Shull 2008). During this step, we assume that the 

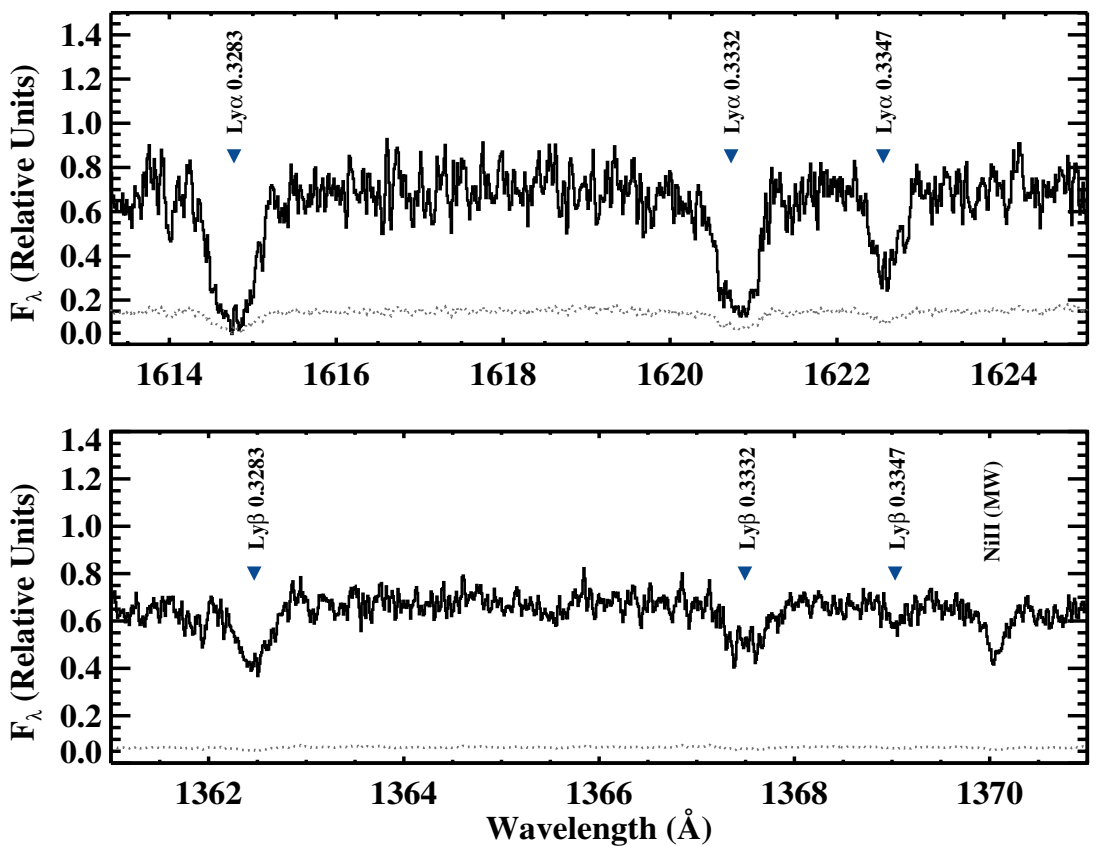

Figure 2. Detail of the COS spectrum of 3C 66A in the regions where we identify Ly $\alpha$ (top) and corresponding Ly $\beta$ (bottom) absorption lines for three gas clouds at $z_{\text {abs }} \sim 0.3283,0.3333$, and 0.3347. Absorption associated with Galactic Ni II is also labeled in the bottom panel.

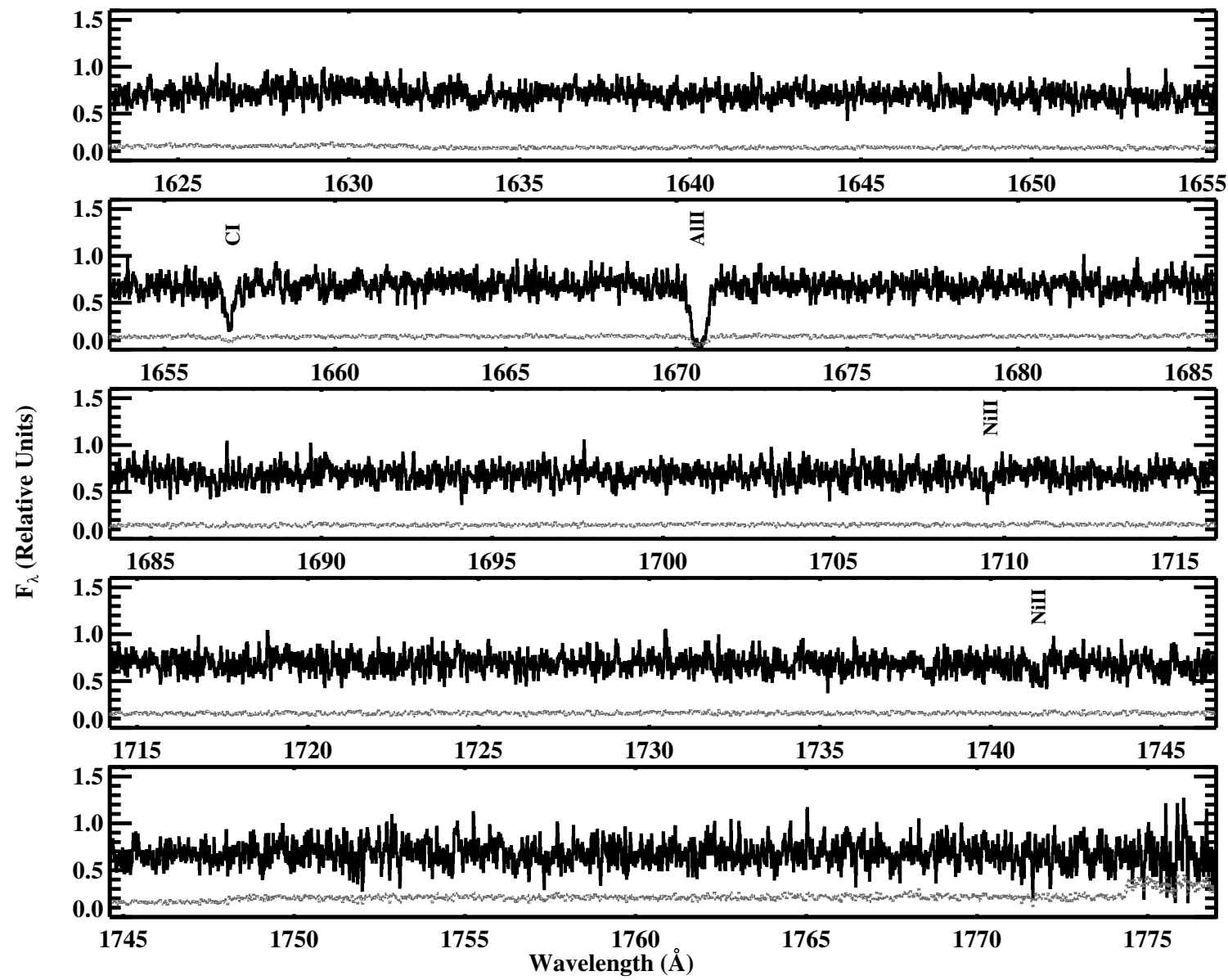

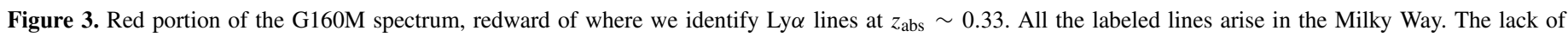
absorption of extragalactic origin places the redshift lower limit of $3 \mathrm{C} 66 \mathrm{~A}$ at $z_{\text {blazar }} \geqslant 0.3347$.

Doppler parameter is not correlated with the equivalent width of the line. Given a line equivalent width, its redshift, and a Doppler parameter, we compute the observed limiting equivalent width $W_{\text {lim }}$ (at $5 \sigma$ ) using the formalism developed for COS spectra by Keeney et al. (2012) and we record only those lines which would be detected in the observed COS spectrum. Note that this procedure naturally accounts for "shadowing" due to Milky Way absorption lines. 

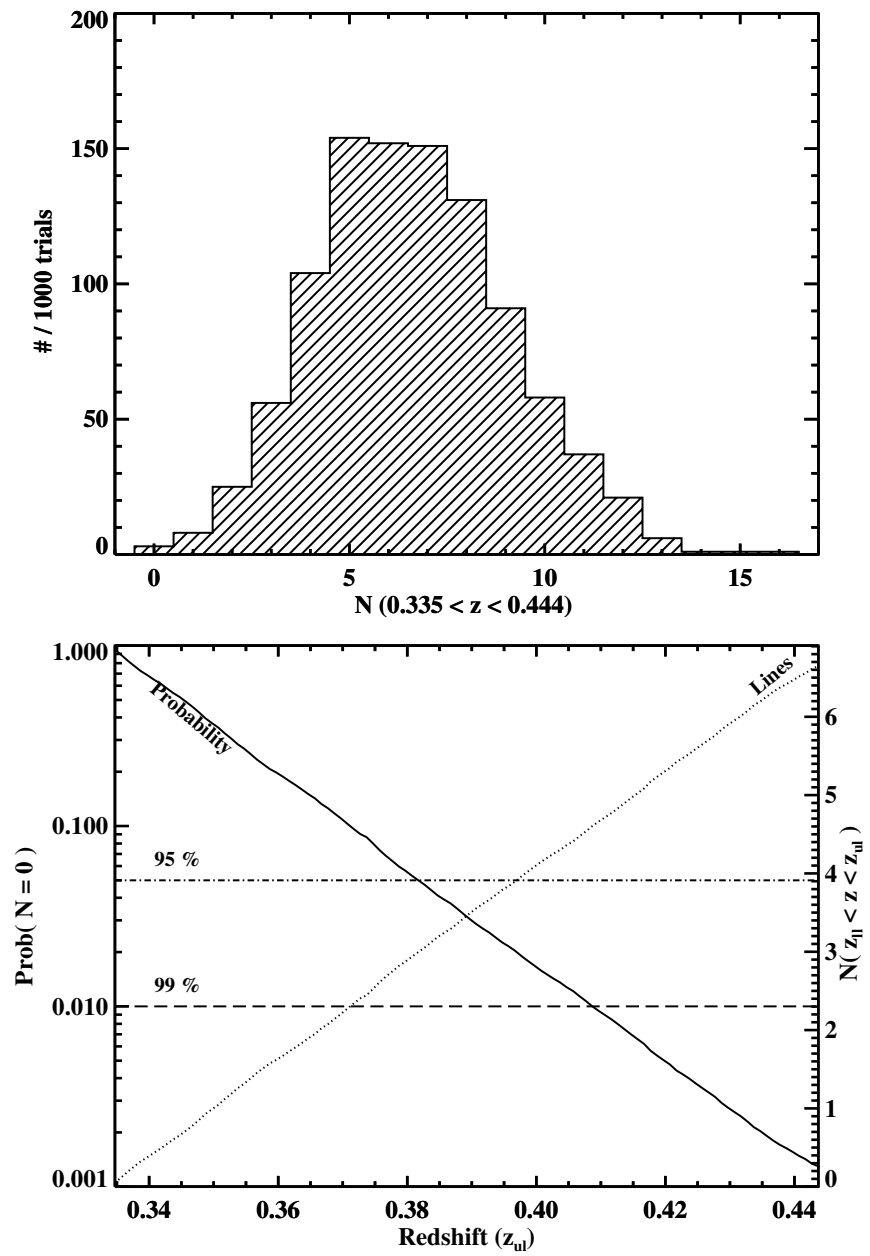

Figure 4. Top: distribution of the number of lines detected in 1000 mock spectra for $0.335<z<0.444$. Bottom: the probability to observe no Ly $\alpha$ lines if 3C 66A lies beyond $z_{\mathrm{ul}}$ given the expected number of Ly $\alpha$ lines in the redshift interval $z_{\mathrm{ll}}<z<z_{\mathrm{ul}}$ derived from Monte Carlo simulations (dotted line).

The top panel of Figure 4 shows the number of intervening absorption lines detected in 1000 mock spectra within the redshift interval $0.335 \lesssim z \lesssim 0.444$. According to this figure, we should expect to detect $\sim 5$ or more lines if $3 \mathrm{C} 66 \mathrm{~A}$ lies at $z_{\mathrm{ul}}>0.444$, and, although realizations with no lines are possible, they are extremely rare ( $<1 \%$ of the total trials). Under the simplistic assumption that the number of absorption lines is not correlated in velocity space, the mock realizations shown in the top panel of Figure 4 follow a Poisson distribution. Therefore, we can adopt Poisson statistics to express the probability of finding no detected lines between $z_{\mathrm{ll}}$ and $z_{\mathrm{ul}}$, given a typical number of Lyman forest lines in that redshift interval $N\left(z_{11}<z<z_{\mathrm{ul}}\right)$.

As shown in the bottom panel of Figure 4, the expected number of absorption lines increases proportionally to the redshift interval $\Delta z=z_{\mathrm{ul}}-z_{11}$ and the probability of finding no absorption lines $P(N=0)$ exponentially decreases with redshift. At $z_{\mathrm{ul}} \sim 0.41, P(N=0) \sim 0.01$, and therefore we conclude that $3 \mathrm{C} 66 \mathrm{~A}$ is likely to lie between $0.3347<$ $z_{\text {blazar }} \lesssim 0.41$. We can further rule out $z_{\text {blazar }} \gtrsim 0.444$ based on the fact that $P(N=0) \sim 0.001$ for $z_{\mathrm{ul}} \sim 0.444$. We note that consistent probabilities can be recovered directly from the Monte Carlo simulations, without explicitly using Poisson statistics. However, it should be noted that our Monte Carlo simulations do not include correlated absorption systems in the Ly $\alpha$ forest. Further, this calculation does not account for mechanisms that could enhance (e.g., galaxy clustering) or suppress (photoionization along the line of sight) the incidence of Ly $\alpha$ lines in proximity to a blazar compared to the mean value observed in the IGM, although there is no evidence for highly ionized gas (i.e., $\mathrm{N} v$ absorption) at $z \sim 0.335$. With proximity effects included, the predicted limits are subject to $\sim 1000 \mathrm{~km} \mathrm{~s}^{-1}$ uncertainty (i.e., $\sim 0.003$ in redshift space). Notably, there have been previous suggestions that $3 \mathrm{C} 66 \mathrm{~A}$ is a member of a cluster at $z \sim 0.37$ (Butcher et al. 1976; Wurtz et al. 1993, 1997).

The limits placing the redshift between 0.3347 and 0.41 disfavor the past tentative measurements of $z=0.444$ by Miller et al. (1978) and Kinney et al. (1991), both of which were based on the measurement of single, weak lines. The limits derived from the COS observations are, however, in good agreement with other past estimates of the blazar distance. Finke et al. (2008) set a lower limit of $z \geqslant 0.096$, an estimation based on the expected equivalent widths of absorption features in the blazar host galaxy, while a distance estimate of $z \simeq 0.321$, noting a large error, was formed based on the assumption that host galaxies of a blazars could be taken as standard candles. An estimate for the blazar redshift of $z=0.34 \pm 0.05$ was found by Prandini et al. (2010), who extracted the approximate redshift by correcting the $\mathrm{TeV}$ spectrum of the blazar for EBL absorption to match the index measured by the Fermi Large Area Telescope (LAT; Atwood et al. 2009), most sensitive to gamma rays between $300 \mathrm{MeV}$ and $\sim 100 \mathrm{GeV}$ which are largely unaffected by the EBL. The redshift limits for $3 \mathrm{C} 66 \mathrm{~A}$ are also in good agreement with a recent EBL modelindependent study of the gamma-ray horizon, as determined by synchrotron self-Compton modeling of VHE blazar broadband spectra (Dominguez et al. 2013).

\section{ABSORPTION OF VERY HIGH ENERGY GAMMA-RAYS FROM 3C 66A}

The energy- and redshift-dependent absorption of gamma rays by the EBL can be estimated using model-specific optical depths, $\tau(E, z)$, where the intrinsic flux $\left(F_{\text {int }}\right)$ can be estimated by the observed flux $\left(F_{\text {obs }}\right)$ using the relation $F_{\text {int }} \sim$ $F_{\text {obs }} \times e^{\tau(E, z)}$. The intrinsic index of a blazar can be used to estimate the spectral properties of the EBL under the physically motivated assumption that the intrinsic spectrum of a source undergoing Fermi shock acceleration, characterized by the powerlaw $d N / d E \propto E^{-\Gamma}$, cannot be harder than $\Gamma=1.5$. If the intrinsic VHE spectrum is significantly harder than the $\Gamma=1.5$ limit, it can be argued that the gamma-ray opacity of the EBL model which was used for deabsorption is too high. The index limit of 1.5 is derived from the standard leptonic and hadronic emission scenarios used to describe blazar non-thermal emission. This limit is also in agreement with the hardest gamma-ray index reported by Fermi-LAT for a blazar (Nolan et al. 2012). The indices for sources derived from photons with energies of less than $100 \mathrm{GeV}$ are not significantly affected by EBL absorption and so reflect the intrinsically emitted spectra of blazars in the high energy gamma-ray band.

Under the assumption that blazars do not harden with increasing energy, EBL flux constraints are also possible by comparing deabsorbed VHE spectra to the extrapolations based on the LAT-measured spectral indices. Using this method, the Fermi and VERITAS indices measured during a state of elevated flux from $3 \mathrm{C} 66 \mathrm{~A}$ in October of $2009\left(\Gamma=1.8 \pm 0.1_{\text {stat }}\right.$ and $4.1 \pm$ $0.6_{\text {stat }}$, respectively; Abdo et al. 2011) allow the investigation 


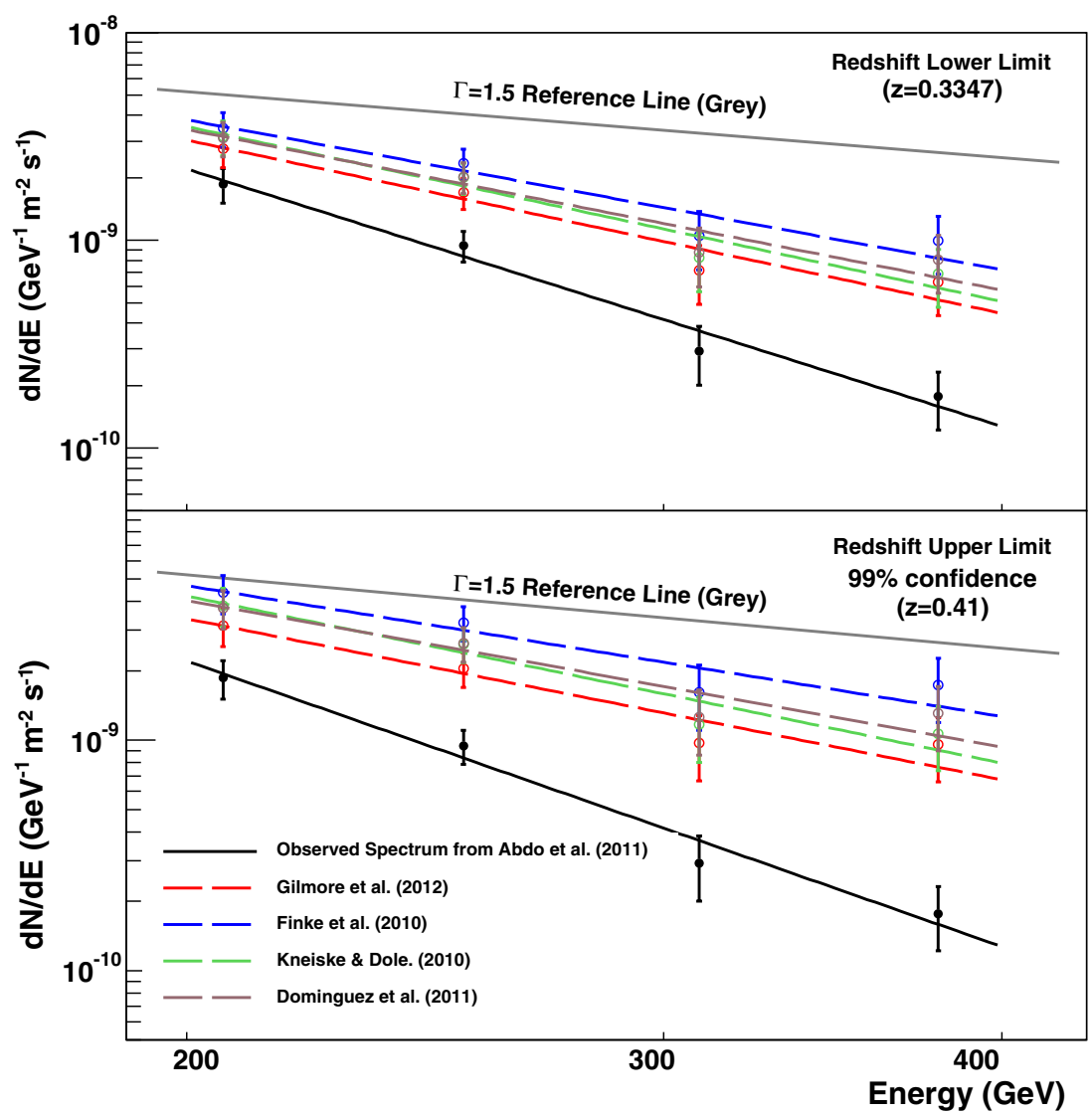

Figure 5. Deabsorbed spectra for 3C 66A for the $z_{11}$ of 0.3347 (top panel) and 99\% confidence level $z_{\mathrm{ul}}$ of 0.41 (bottom panel), where the observed VHE spectrum (black solid line) is taken from Abdo et al. (2011), with an index of $\Gamma=4.1 \pm 0.6_{\text {stat }}$ for the applied differential power-law of the form $d N / d E=\left(E / E_{o}\right)^{-\Gamma}$. For reference, a spectrum with an index of $\Gamma=1.5$ is shown as the theoretical limit for an intrinsic index, as explained in the text. The resulting indices for each redshift and model are summarized in Table 1.

(A color version of this figure is available in the online journal.)

Table 1

Intrinsic Indices $(\Gamma)$ Resulting from the Deabsorption of the VERITAS Observed Spectrum Reported in Abdo et al. (2011)

\begin{tabular}{lcc}
\hline \hline EBL & $\begin{array}{c}\text { Deabsorbed } \\
\text { Index }\end{array}$ & $\begin{array}{c}\text { Deabsorbed } \\
\text { Model }\end{array}$ \\
Used & $z=0.3347$ & $z=0.41$ \\
\hline Gilmore et al. (2012) & $2.8 \pm 0.6$ & $2.3 \pm 0.6$ \\
Finke et al. (2010) & $2.4 \pm 0.6$ & $1.9 \pm 0.6$ \\
Kneiske \& Dole (2010) & $2.8 \pm 0.6$ & $2.4 \pm 0.6$ \\
Dominguez et al. (2011) & $2.6 \pm 0.6$ & $2.1 \pm 0.6$ \\
\hline
\end{tabular}

Notes. Indices are calculated by taking the VERITAS-measured differential flux and flux errors and multiplying by $e^{\tau}$, where $\tau$ is an energy and redshift dependent optical depth taken from the EBL models. The resulting flux in each bin is then fit with the differential power-law of the form $d N / d E=\left(E / E_{o}\right)^{-\Gamma}$, where $E_{o}$ is $250 \mathrm{GeV}$.

of possible constraints on the EBL density, pending a reliable distance measurement. Previously, the deabsorption of the VHE spectrum of $3 \mathrm{C} 66 \mathrm{~A}$ has been completed with the uncertain spectroscopic redshift of $z=0.444$ (e.g., Finke et al. 2010; Dominguez et al. 2011; Aleksić et al. 2011). Notably, Gilmore et al. (2012) show that the intrinsic spectrum derived from deabsorption of $3 \mathrm{C} 66 \mathrm{~A}$ with the tentative redshift of $z=0.444$ is the hardest of the deabsorbed VHE BL Lacertae objects.

Figure 5 shows the VERITAS-measured VHE spectrum of the blazar 3C 66A from Abdo et al. (2011) (black solid line) when deabsorbed for the redshift upper and lower limits from this work. These deabsorbed spectra are calculated by multiplying the measured differential flux values by $e^{\tau(E, z)}$ for various EBL models. The resulting intrinsic flux estimates are then refit with a differential power-law for the redshift lower limit (top) and 99\% upper limit (bottom). The fitted intrinsic indices for both the lower and upper limits on redshift are summarized in Table 1. The hardest deabsorbed spectra result from the Finke et al. (2010) EBL model, but all fitted power-laws provide indices softer than the $\Gamma=1.5$ limit (shown for reference in Figure 5 by the gray solid line at a comparable normalization to the deabsorbed spectra). The resulting indices are also below the Fermi-LAT measured index of $\Gamma=1.8 \pm 0.1$.

\section{CONCLUSION}

Observation of the $z \sim 0$ Ly $\alpha$ forest in the direction of the 3C 66A with HST/COS provides a direct lower and statistical upper redshift limit for the blazar. The detection of three clouds at $z_{\text {abs }} \sim 0.3283,0.3333$, and 0.3347 provide the $z=0.3347$ lower limit on the blazar redshift. Assuming that the incidence of Lyman absorption systems is a Poisson distribution in $z$, we can conclude that the blazar is likely to lie $z_{\text {blazar }} \lesssim 0.41(99 \%$ confidence level) and exclude a $z \geqslant 0.444$ at $99.9 \%$.

Based on the assumption that the intrinsic index cannot be harder than $\Gamma=1.5$, the redshift limits derived from the FUV observations do not place the blazar at a sufficient distance to utilize the observed VHE spectrum during an elevated state in 2009 October to constrain the EBL density. Moreover, the 
distance is not sufficient to extract an upper limit on the EBL density based on the similar assumption that the intrinsic VHE index is not harder than the Fermi observed index.

We are grateful to Robert da Silva for insightful discussions on the statistical analysis of these data. Support for program HST-GO-12863 and for Hubble Fellow M.F. (grant HF-51305.01-A) were provided by NASA awarded through grants provided by the Space Telescope Science Institute, which is operated by the Association of Universities for Research in Astronomy, Inc., for NASA, under contract NAS 5-26555. Additional support for this work came from National Science Foundation award PHY-0970134. C.D. was supported by NASA grants NNX08AC146 and NAS5-98043 to the University of Colorado at Boulder.

Facility: HST (COS)

\section{REFERENCES}

Abdo, A. A., Ackermann, M., Ajello, M., et al. 2011, ApJ, 726, 43

Aharonian, F., Akhperjanian, A. G., Bazer-Bachi, A. R., et al. 2006, Natur, 440, 1018

Albert, J., Aliu, E., Anderhub, H., et al. 2008, Sci, 320, 1752

Aleksić, J., Antonelli, L. A., Antoranz, P., et al. 2011, ApJ, 726, 58

Atwood, W., Abdo, A., Ackermann, M., et al. 2009, ApJ, 697, 1071
Bramel, D. A., Carson, J., Covault, C. E., et al. 2005, ApJ, 629, 108

Butcher, H., Oemler, A., Tapia, S., \& Tarenghi, M. 1976, ApJL, 209, L11

Danforth, C. W., Keeney, B. A., Stocke, J. T., Shull, J. M., \& Yao, Y. 2010, ApJ, 720, 976

Danforth, C. W., Nalewajko, F., \& Keeney, B. A. 2013, ApJ, 764, 57

Danforth, C. W., \& Shull, J. M. 2008, ApJ, 679, 194

Dominguez, A., Finke, J., Prada, F., et al. 2013, ApJ, submitted

Dominguez, A., Primack, J., Rosario, D. J., et al. 2011, MNRAS, 410, 2556

Finke, J., Razzaque, S., \& Dermer, C. 2010, ApJ, 712, 238

Finke, J., Shields, J., Böttcher, M., \& Basu, S. 2008, A\&A, 477, 513

Ghavamian, P., Aloisi, A., Lennon, D., et al. 2010, BAAS, 42, 499

Gilmore, R., Madau, P., Primack, J., et al. 2009, MNRAS, 399, 1694

Gilmore, R., Somerville, R., Primack, J., \& Dominguez, A. 2012, MNRAS, 422, 3189

Hauser, M., \& Dwek, E. 2001, ARA\&A, 39, 249

Keeney, B. A., Danforth, C. W., Stocke, J. T., France, K., \& Green, J. C. 2012, PASP, 124, 830

Kinney, A. L., Bohlin, R. C., Blades, J. C., \& York, D. G. 1991, ApJS, 75, 645

Kneiske, T. M., \& Dole, H. 2010, A\&A, 515, 19

Mazin, D., \& Raue, M. 2007, A\&A, 471, 439

Miller, J. S., French, H. B., \& Hawley, S. A. 1978, in Pittsburgh Conf. BL Lac Objects, ed. A. M. Wolfe (Pittsburgh, PA: Univ. Pittsburgh), 176

Nolan, P. L., Abdo, A. A., Ackermann, M., et al. 2012, ApJS, 199, 31

Orr, M., Krennrich, F., \& Dwek, E. 2011, ApJ, 733, 77

Penton, S. V., Stocke, J. T., \& Shull, J. M. 2004, ApJS, 152, 29

Prandini, E., Bonnoli, G., Maraschi, L., Mariotti, M., \& Tavecchio, F. 2010, MNRAS, 405, 76

Wurtz, R., Ellingson, E., Stocke, J., \& Yee, H. 1993, AJ, 106, 869

Wurtz, R., Stocke, J., Ellingson, E., \& Yee, H. 1997, ApJ, 480, 547 